\title{
Social Attributes Analysis based on Real Trajectory of Vehicles
}

\author{
Peng Zhou ${ }^{1, a}$, Xiaoqiang Xiao ${ }^{2, b}$ and Weixun Ning ${ }^{3, c}$ \\ ${ }^{1}$ National University of Defense Technology, Changsha, China \\ ${ }^{2}$ National University of Defense Technology, Changsha, China \\ ${ }^{3}$ National University of Defense Technology, Changsha, China \\ azhoupeng11@nudt.edu.cn, bxqxiao@nudt.edu.cn, 'ningweixun@nudt.edu.cn
}

\begin{abstract}
Keywords: social attribute; VANET, trajectory; centricity; preference
Abstract. Social attributes are helpful to improve the performance of network including all kinds of wireless networks. How to discover the social attributes of vehicles trajectory becomes a challenge problem for VANET. Trying to find out the social relationship from the real trajectory becomes an attractive idea. This article refers to the concept centricity and preferences in the social network, obtains the social attributes of the vehicles by analyzing and processing the real trajectory data. The performance of communication can be improved by taking advantage of these attributes because of the better choice of relay nodes.
\end{abstract}

\section{Introduction}

With the popularity of vehicles, the VANET (Vehicular Ad-hoc Network) has been studied further, but there are few studies of vehicle social attributes. Because the drivers of the vehicles are human beings, the movement characteristics of vehicles will inevitably have the properties of social networks. Therefore, the analysis of the social attributes of vehicles will have a great help for studying the VANET. For example, during the routing algorithm design, the social attributes can guide the choice of the relay node.

The concepts of centricity and preference have been proposed in social networks [1], and they are widely used in opportunistic routings. Our basic idea is to extend these concepts into VANET and the social attributes of vehicles can be obtained by analyzing the real trajectories.

At first, this paper shows some relative works about the researches of trajectory and the application of social attributes in routings. Then we give the definitions of centricity and preference for the vehicles. After that, through the analysis of the trajectories, the centricities and preferences of the vehicles are drew out. Finally, we give the conclusion that the social attributes got from the trajectories can be used to improve the performance of routing by choosing a better relay node.

\section{Relative Works}

The current researches on the vehicle trajectory can be grouped into three categories: social dynamics, traffic dynamics and operational dynamics [2]. Social dynamics is defined as the work studying the collective behavior of a city's population, find the "hottest" spots around a city which means the place people are interested in; Traffic dynamics studies the resulting flow of the population through the road network, most of these work generally aim at predicting the traffic conditions and can be useful for providing real-time traffic indicators and travel time estimation for drivers; Operational dynamics refers to the general study and analysis of taxi drivers' modus operandi, its aim is to be able to learn from taxi drivers' excellent knowledge of the city, as well as to detect abnormal behaviors.

The applications of social attributes in opportunistic network are mainly improving routing algorithm, some protocols are proposed by analyzing the social attributes of the vehicles in the network, predicting the encounter and movement information of vehicles. The packet delivery ratio and overhead of these routing algorithms are always cared about.

BubbleRap is a forwarding protocol which proposes a distributed method to detect communities; it uses the centrality metric as a rank for each node. A node which has a message bubbles this 
message to the node with a higher rank [3]. SimBet uses centricity and social similarity metrics to define the probability of the node contacting the destination [4]. PeopleRank proposes a scheme to rank nodes based on its "importance' in the network based on the idea of page rank to index web pages on Google [5]. Considering the cost and some other factors, most of the routing protocols are proposed through analysis of social behavior and are evaluated by simulation. Only a few protocols are proposed based on the analysis of real trajectory, 3R [6] is a protocol characterized the trace as a fine-grained encounter trace showing that the pattern of those encounters is regular and predictable.

To the best of our knowledge, for many researches of vehicle trajectories, there are few of them help to improve the performance of communication. Meanwhile, the social attributes on which many routing algorithms based are not excavated from real vehicle trace [7]. In order to take advantages of the real social relationship among vehicles, the VANET routing algorithm considering the social attributes should analyze the real vehicle trajectories [8].

\section{The Analysis of Trajectory for Social Attribute}

The trajectory data contains the state information such as the location of a node at a certain moment. As for the vehicle trajectory data, for example, they include license plate number, sampling time, latitude, longitude, speed, direction and so on. To get some social attributes, the data should be preprocessed to extract the needed information, and can be used for further analyze [9].

Analysis of Vehicular Centricity. A node's centricity is associated with the number of nodes it encountered. The centricity of a node is the ratio of the number of nodes it encountered and the total number of nodes except itself in the network. As shown in Eq.1.

$$
C_{k}=\frac{w_{h}}{n-1}
$$

$C_{k}$ is the centricity of a node, $n$ is the total numbers in the network, $e_{k}$ is the number of nodes the node $k$ encountered. When a node could send messages to another, this situation is called encounter. That is, two nodes encounter only if their distance is less than the range of communication. However, the problem of trajectory is that the sampling times are different for different nodes. So it's necessary to calculate the distance of two nodes at the middle of two sampling times.

As shown in Fig 1, $\mathrm{N}_{1}$ and $\mathrm{N}_{2}$ are the position of two nodes at $\mathrm{t}_{1}$ and $\mathrm{t}_{2}$ respectively; the nodes' speeds are $v_{1}$ and $v_{2}$. The circles are nodes' communication range. Assuming that time $t_{1}$ is earlier than $\mathrm{t}_{2}$, we get $\Delta t$ which equals to $\mathrm{t}_{2}$ minus $\mathrm{t}_{1} . \mathrm{N}_{1}$ ' and $\mathrm{N}_{2}$ ' is the position of the nodes at the moment $\left(t_{1}+t_{2}\right) / 2, d$ is the distance of two nodes at that moment, when the value of $d$ is less than the node's communication radius $r$, two nodes encounter.

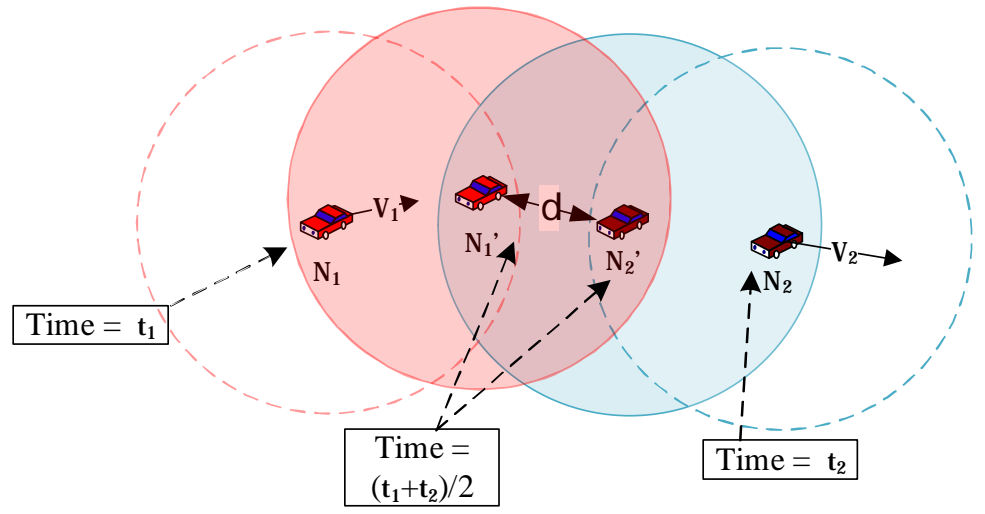

Fig. 1 the schematic diagram of nodes encounter

To make the calculation more accurate, $\Delta t$ should be limited to a small value. Assuming that the sampling time interval of trajectory data is $\Delta T$, so from time $\mathrm{T}-\Delta T / 2$ to $\mathrm{T}+\Delta T / 2$, the speed of the node could approximately be regard as the speed at time T. So, $\Delta t$ should be smaller than $\Delta T / 2$. If the nodes' coordinates are $\left(\mathrm{x}_{1}, \mathrm{y}_{1}\right)$ and $\left(\mathrm{x}_{2}, \mathrm{y}_{2}\right)$, the condition of encounter is shown in Eq.2. 


$$
\left\{\begin{array}{c}
r>\sqrt{\left(\left(x_{1}-x_{2}\right)+\Delta t / 2 *\left(v_{1}-v_{2}\right)\right)^{2}+\left(\left(y_{1}-y_{2}\right)+\Delta t / 2 *\left(v_{1}-v_{2}\right)\right)^{2}} \\
\Delta t<\Delta T / 2
\end{array}\right.
$$

According to the judgment condition of encounter, the number of nodes a vehicle encountered can be drawn out by analyzing the trajectories, then the centricity can be calculated.

The Analysis of Vehicular Preference. The preference of vehicle is different from the "hottest" spot, it show the area where the vehicle prefer to appearing in. As shown in Fig.2, the points are the positions of a vehicle recorded for a long time, and the circle is the area with highest density, which means the vehicle prefer to moving around in that area. So the preference can be represented by the circle D which is shown as Eq.3, where the radius of circle is $\mathrm{R}$ and the center of circle is $\mathrm{O}$.

$$
\mathrm{D}=(\mathrm{R}, \mathrm{O})
$$

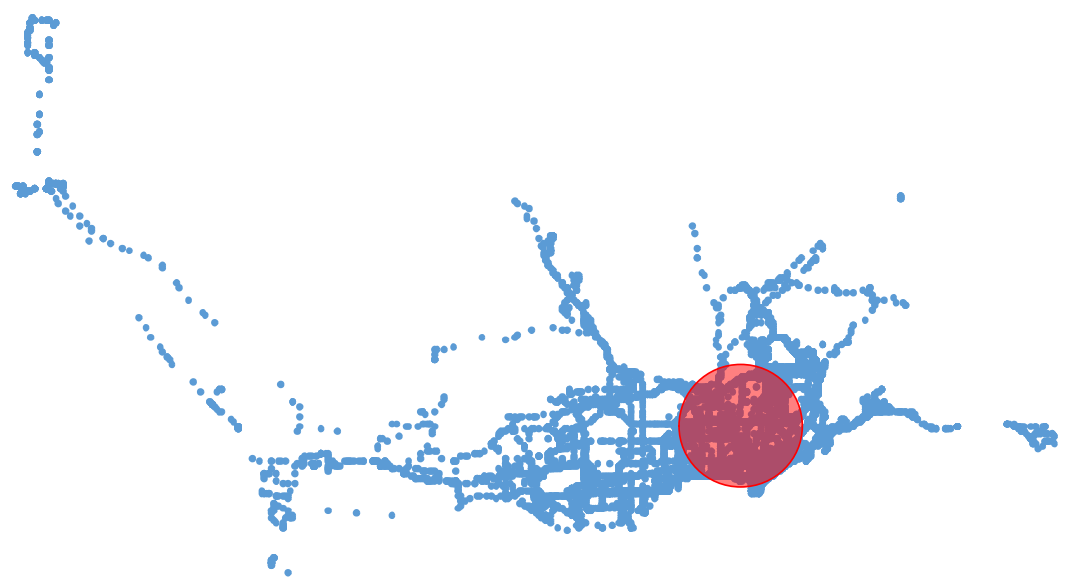

Fig. 2 the schematic diagram of node's preference

The radius $\mathrm{R}$ is decided by the average velocity of the vehicle. It is easy to calculate the average velocity, and $\mathrm{R}$ is equal to the distance the vehicle run at that velocity in an unit time, without loss og generality, assume the unit time is half an hour. After that, the center of circle should be ascertained. As shown in Eq.4, $d_{k}$ is the density of the circle whose center is point $k$ and radius is $\mathrm{R}$, then $\mathrm{O}$ can be located by the equation.

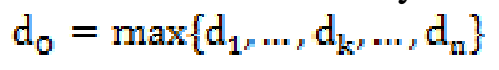

\section{The Result of Experiment}

The original data of the our experiment is the GPS trajectories of more than 400 taxis. Each data element records the ID of the vehicle, the sampling time, the longtitude, the langtitude, the velocity, the status and the angle of vehicle moving. According to the methods refered to in the last section, the centricitties and preferences can be calculated. The results are shown in Fig.3 and Fig.4, which are drawn by the softwave EXCEL.

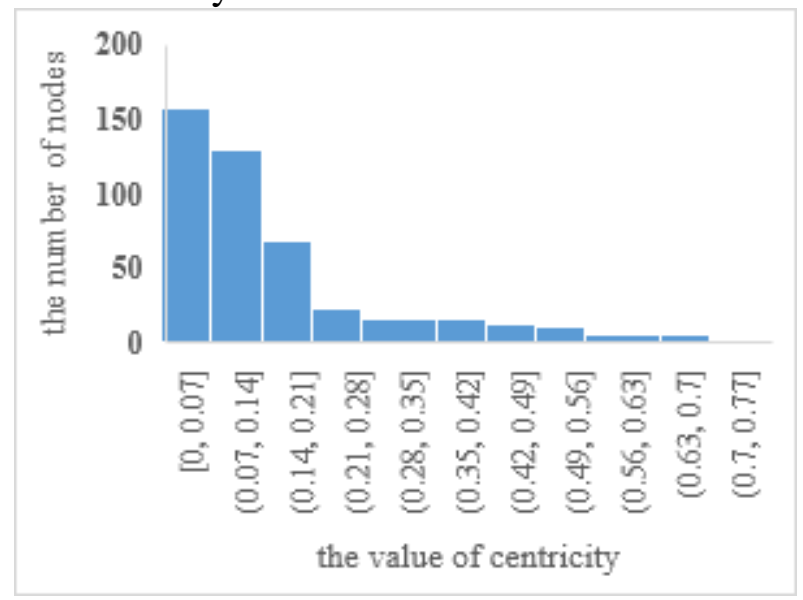

Fig. 3 the distribution of centicities

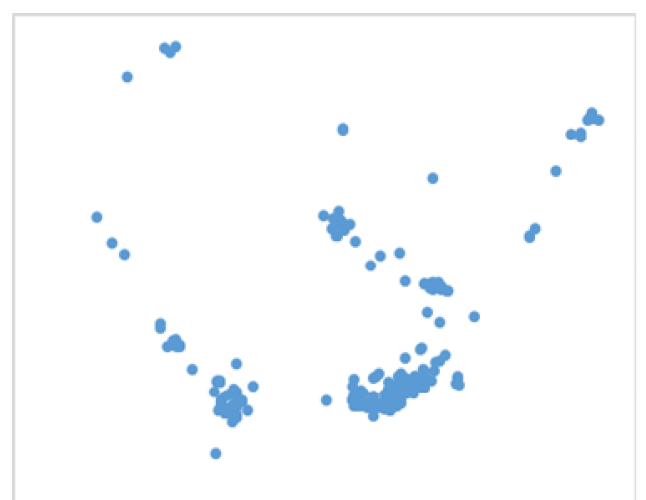

Fig.4 the distribution of the center of preference area 
It can be seen from Fig.3, most of the nodes have low centricities, choosing a node with higher centricity as a relay node would be better then choosing randomly during routing algorithn design. From Fig.4, we can see there are several cluster in the distribution of center of preference area. When two node with high overlap rate of the preference area, the encounter probability of these two vehicles will also be high.

These attributes can be used to improve the performance of the VANET routing algorithm [10]. Let's take the BubbleRap algorithm as an example. During the progress of forwarding, the next hop should be the node whose centricity value is higher, or the node which have higher overlap rate with the destination in preference.

\section{Conclusions}

This paper focuses on the centricities and preferences of vehicles by analyzing the vehicle trajectory data. First, the centricity and the preference were redefined which are suitable for the vehicles; Then the programs are code based on the definitions, and the results were shown after ran the programs whose input is the original data. The improvement of routing is predictable.

Besides the centricity and preference, there are some other social attributes such as the relationship between different nodes and the communities of vehicles. Different routing algorithms based on social attributes may have different performance, so the simulations of algorithms are necessary.

\section{Acknowledgements}

This work is financially supported by the National Science Foundation of China under Grant No.61272485.

\section{References}

[1]. Stanley W, Katherine F. Social Network Analysis: Methods and Applications [M]. (In Chinese) Beijing: China Renmin University Press, 2012

[2]. Castro P S, Zhang D, Chen C, et al. From taxi GPS traces to social and community dynamics: A survey[J]. Acm Computing Surveys, 2014, 46(2):1167-1182.

[3]. Hui P, Crowcroft J, Yoneki E. BUBBLE Rap: Social-Based Forwarding in Delay-Tolerant Networks[J]. IEEE Transactions on Mobile Computing, 2011, 10(11):1576-1589.

[4]. E. Daly, M. Haahr, Social network analysis for routing in disconnected delay-tolerant MANETs[C]// Proceedings of the 8th ACM International Symposium on Mobile Ad Hoc Networking and Computing, ACM, 2007:32-40.

[5]. Mtibaa, M. May, C. Diot, M. Ammar, Peoplerank: social opportunistic forwarding[C] //Proceedings of IEEE INFOCOM, IEEE, 2010:1-5.

[6]. Long V, Do Q, Nahrstedt K. 3R: Fine-grained encounter-based routing in Delay Tolerant Networks[J]. IEEE International Symposium on a World of Wireless Mobile \& Multimedia Networks, 2011:1-6.

[7]. Bonola M, Bracciale L, Loreti $\mathrm{P}$, et al. Opportunistic communication in smart city: Experimental insight with small-scale taxi fleets as data carriers[J]. Ad Hoc Networks, 2016, 43:43-55.

[8]. Mota V F S, Cunha F D, Macedo D F, et al. Protocols, mobility models and tools in opportunistic networks: A survey[J]. Computer Communications, 2014, 48(8):5-19.

[9]. Pagani E, Valerio L, Rossi G P. Weak social ties improve content delivery in behavior-aware opportunistic networks[J]. Ad Hoc Networks, 2015, 25(PB):314-329.

[10]. Liu T, Zhu Y, Jiang R, et al. A sociality-aware approach to computing backbone in mobile opportunistic networks[C]// Global Communications Conference. IEEE, 2013:46-56. 